\title{
COMPACT 200-keV ELECTRON BEAM SYSTEMS
}

\author{
V. Pirozhenko*, BioSterile Technology, Inc., Fort Wayne, USA \\ A. Korolev, K. Simonov, "Istok", Frjazino, Russia
}

\begin{abstract}
The systems are intended for material radiation processing by accelerated electron beams. The beam is generated by unique sealed-off electron gun providing good reliability and small bulk of the system. The beam is extracted to the atmosphere through 20-micron titanium foil and is uniformly distributed over the area of exposure that is $20 \times 10 \mathrm{~cm}$ or $30 \times 4 \mathrm{~cm}$. The systems operate in the pulse mode, with high pulse repetition rate. The systems are compact, they have protective radiation shielding and can be safely operated within any laboratory environment. The systems provide high radiation dose rate (up to $60 \mathrm{kGy} / \mathrm{s}$ ) in thin films and layers. They may be used as research devices in the field of materials investigations, for material treatment, for radiation sterilization, etc.
\end{abstract}

\section{INTRODUCTION}

Development of compact radiation systems is due to the evolution of radiation technologies [1, 2]. The systems are intended for radiation processing of thin films and material surfaces by the accelerated electron beams. Electrons energies of 100 to $200 \mathrm{keV}$ are most suited for the compact systems. On the one hand, the electron beam can pass well through foils in the output windows starting with $100 \mathrm{keV}$ energy. On the other hand, at $200 \mathrm{keV}$ energy the depth of the electron penetration is large enough for a number of technologies.

Two types of compact systems are presented in the paper. The first type (PYXIS) is intended for the irradiation of fixed objects, the second type (TAPIS) - for the irradiation of continuously moving tape. Preliminary results of PYXIS prototype development had been reported earlier [3].

\section{PERFORMANCE CHARACTERISTICS}

The compact systems are based on the usage of sealed-off electron guns. The systems have the following general characteristics:

- objects are irradiated by electron beams with energies up to $200 \mathrm{keV}$ penetrating to materials $0.1-0.2 \mathrm{~mm}$;

- irradiation is performed by a wide electron beam distributed over the area of exposure;

- irradiation takes place in nitrogen to eliminate the effect of oxygen on the processing;

- the systems operate in pulse mode with low pulse duration and high pulse repetition rates;

- the major parameters of the systems are variable within broad ranges that is convenient for experimental works.

* E-mail: v_pirojenko@mail.ru
Table 1. Characteristics of the systems

\begin{tabular}{|l|c|c|}
\hline \multicolumn{1}{|c|}{ System } & PYXIS & TAPIS \\
\hline Accelerating voltage $(\mathrm{kV})$ & $100-200$ & $100-200$ \\
\hline Maximum dose rate $(\mathrm{kGy} / \mathrm{s})$ & 30 & 60 \\
\hline Pulse dose rate $(\mathrm{kGy} / \mathrm{s})$ & $10^{5}$ & $2.6 \times 10^{5}$ \\
\hline Pulse repetition rate $(\mathrm{pps})$ & $5-300$ & $5-150$ \\
\hline Beam pulse duration $(\mu \mathrm{s})$ & 1 & 1.5 \\
\hline Irradiation area $(\mathrm{mm})$ & $200 \times 100$ & $300 \times 40$ \\
\hline
\end{tabular}

\section{ELECTRON GUNS}

Devised sealed-off electron guns generate and extract to the atmosphere wide electron beams with high pulse power. The guns are fabricated and pumped at the factory, and their operation is similar to that of TV tubes. They are compact, reliable and durable. Usage of the guns makes it possible to form large irradiation area by combining a number of guns located side by side.

Two types of sealed-off electron guns have been developed. The first type contains two round cathodes located at some distance. The second type has a long ribbon cathode.

The electron gun with round cathodes is shown in Fig. 1. The gun has a vacuum casing with two cathodes inside; high-voltage ceramic insulator; output window for the beam extracting to the atmosphere; miniature vacuum pump with permanent magnet.

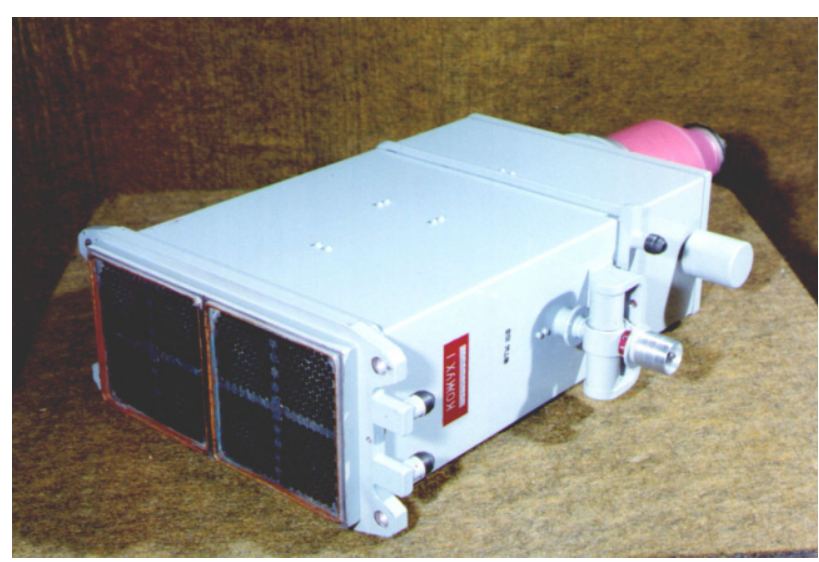

Fig. 1. Sealed-off electron gun

20 micron thick titanium foil is provided at the output window. Two copper grid plates are designed to support the foil and to remove the heat from it. The window is cooled by water running through channels located along the periphery of the window.

Every cathode has a filament and beam forming electrode. The electron optics has been calculated by $3 \mathrm{D}$ code so as to achieve diverging electron beam with 
uniform distribution in the output window. Since the output window has a rectangular form, specially shaped forming electrodes are used.

The beam distribution outside the window differs from that of the incident beam upon the window because of the beam being scattered on the window. If a good uniformity of the beam distribution in the irradiation area is required, it can be achieved by a proper selection of the grid plate perforation diameters. Besides a beam filter with adjusted perforations can be set outside the gun. Distributions of the radiation dose measured along the window (z-axis) are shown in Fig. 2. Non-uniformity of less than $10 \%$ can be obtained by using the filter.

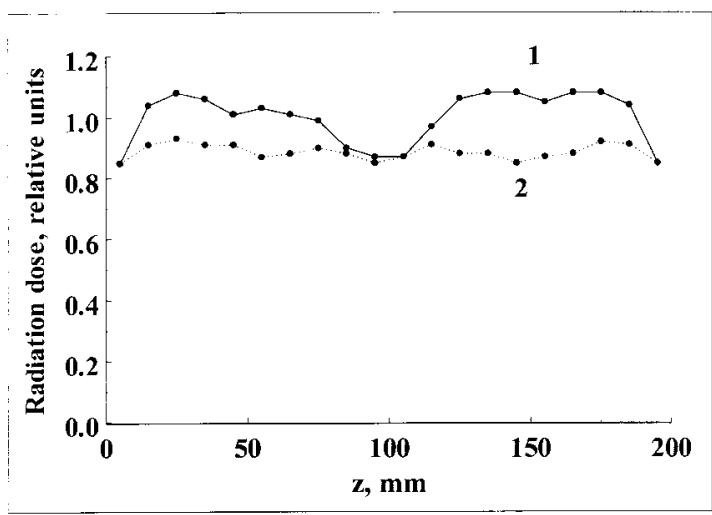

Fig. 2. Measured distributions of the radiation dose:

1 - without filter, 2 - with adjusted filter.

The electron gun with a ribbon cathode has a similar design but is shorter and wider. It contains long ribbon cathode and the forming electrode adequately shaped. The cathode consists of 8 pieces $40 \mathrm{~mm}$ long each. The support grid plate has longitudinal slots $40 \times 4 \mathrm{~mm}$ each.

Developed guns offer high pulse power of electron beam. Several types of the guns have been developed providing a broad beam power range (table 2 ).

Table 2. Parameters of sealed-off electron guns

\begin{tabular}{|l|c|c|c|c|}
\hline \multicolumn{1}{|c|}{ Type of electron gun } & $\begin{array}{c}\text { EG- } \\
1\end{array}$ & $\begin{array}{c}\text { EG- } \\
2\end{array}$ & $\begin{array}{c}\text { EG- } \\
3\end{array}$ & EG-4 \\
\hline Cathode type & \multicolumn{3}{|c|}{ Two round } & Ribbon \\
\hline $\begin{array}{l}\text { Cathode sizes (mm): } \\
\text { diameter } \\
\text { length }\end{array}$ & 5.7 & 11.4 & 20 & 320 \\
\hline $\begin{array}{l}\text { Maximum pulse } \\
\text { current from cathodes } \\
\text { (A) }\end{array}$ & 6 & 15 & 80 & 25 \\
\hline Maximum voltage (kV) & \multicolumn{3}{|c|}{200} & 200 \\
\hline Irradiation area (mm) & \multicolumn{3}{|c|}{$200 \times 100$} & $300 \times 40$ \\
\hline
\end{tabular}

\section{HIGH-VOLTAGE TRANSFORMER}

The high-voltage transformer and the electron gun are combined to form a single structure - irradiator (Fig. 3).

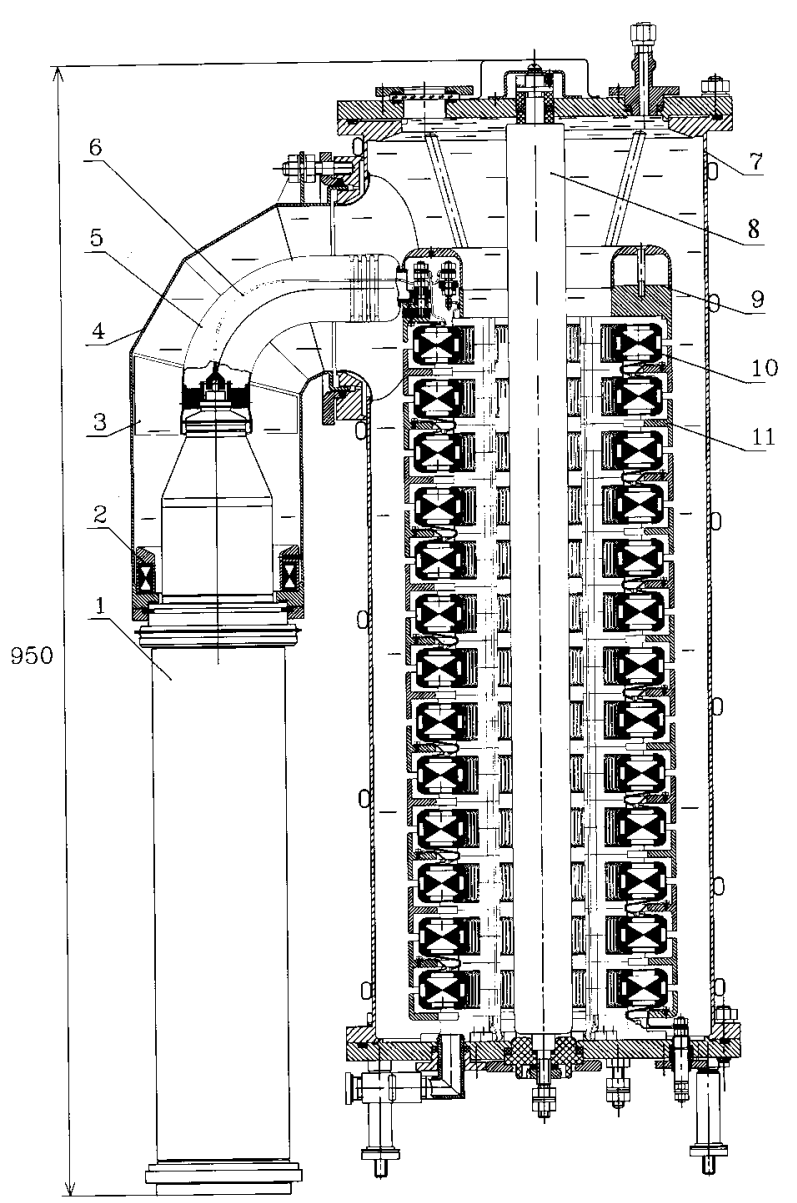

Fig. 3. Design of the PYXIS irradiator.

1 - electron gun, 2 - beam current sensor, 3 - voltage sensor, 4, 5 - external and internal feeder tubes, 6 - filament conductor, 7 - transformer tank, 8 - primary winding, 9, 11 - screens, 10 - transformer module.

The pulse transformer has a modular design. It contains a set of identical modules that are connected in series to form the secondary winding. Each module contains a ring shaped ferromagnetic core, insulating rings and secondary winding. The ferromagnetic core is wound with 20-micron metglass (amorphous alloy) tape, with varnish insulation between the layers. The primary winding of the transformer is a straight conductor on the axis.

The transformer is connected to the electron gun by a short coaxial feeder with voltage and beam current sensors inside. The voltage sensor is a capacitance divider, the beam current sensor is a toroid placed inside an open-ended metal shield. The transformer tank and the feeder are filled with oil so the insulator of the gun is also in the oil.

The devised transformer design and its close connection to the electron gun enables us to develop a high-voltage compact system and obtain a very high transformer voltage ratio that provides a possibility to use a low-voltage pulse modulator. 
An important feature of the systems is low pulse duration. This serves for increasing the allowable intensity of the electric field in the gun and the transformer and developing the compact systems.

Table 3. Parameters of high-voltage pulse transformer

\begin{tabular}{|l|c|}
\hline Number of modules & 13 \\
\hline Transformer voltage ratio & 50 \\
\hline Maximum output pulse voltage $(\mathrm{kV})$ & 240 \\
\hline Tank length $(\mathrm{m})$ & 0.8 \\
\hline Tank diameter $(\mathrm{m})$ & 0.3 \\
\hline
\end{tabular}

\section{SYSTEM DESIGN AND CONTROL}

PYXIS processing unit is shown in Fig. 4. In this system the object is placed in the carriage and inserted under the electron gun, then the irradiation chamber is filled with nitrogen, the electron beam is turned on and irradiates the object being in the fixed position.
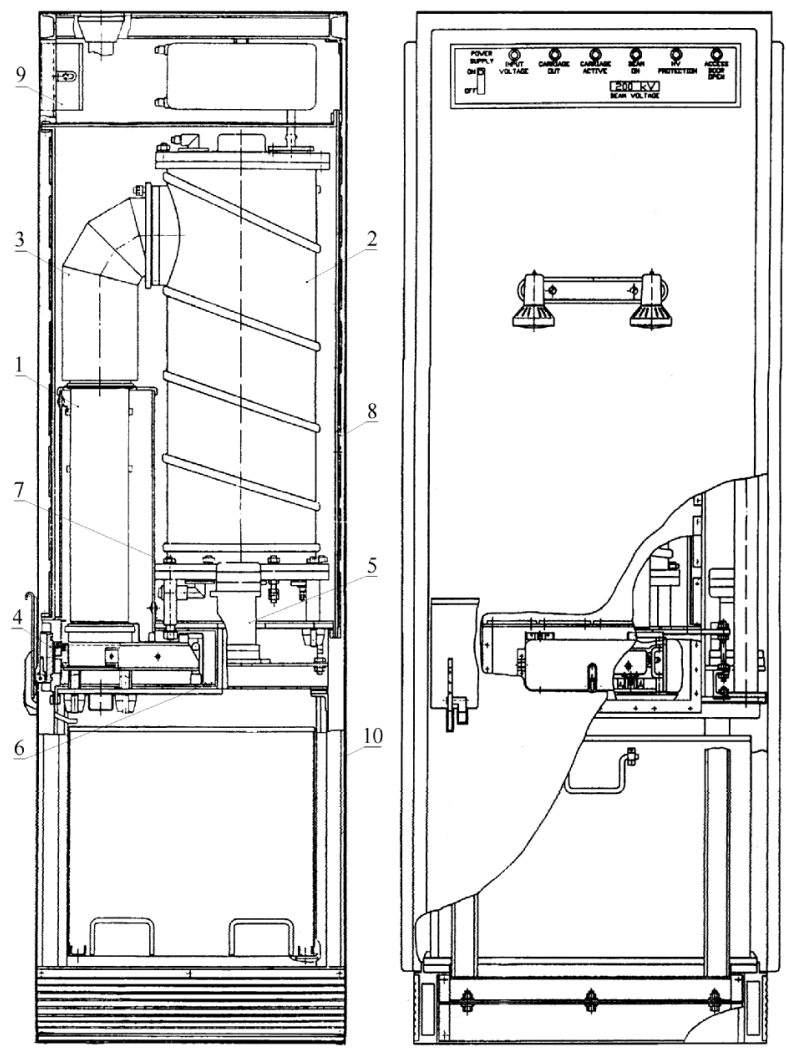

Fig. 4. PYXIS processing unit (side and front views).

1 - electron gun, 2 - transformer, 3 - feeder, 4 - carriage, 5 - carriage drive, 6 - chamber, 7,8 - radiation shielding, 9 - indication panel, 10 - cabinet.

The PYXIS system has two variants: the PYXIS-1 includes power supply blocks located inside the processing unit, the PYXIS-2 includes separate power supply unit with $0.7 \times 0.7 \times 1 \mathrm{~m}$ dimensions.
In TAPIS system, the irradiated $30 \mathrm{~cm}$ wide tape is being moved in the direction perpendicular to the wide side of the electron gun and is rewound from feed to takeup reel. The irradiation chamber is constantly filled with nitrogen. The system includes the power supply unit with $0.7 \times 0.7 \times 2 \mathrm{~m}$ dimensions.

Table 4. Parameters of the systems design

\begin{tabular}{|l|c|c|c|}
\hline \multicolumn{1}{|c|}{ System } & PYXIS-1 & PYXIS-2 & TAPIS \\
\hline Electron gun type & EG-1 & EG-2 & EG-4 \\
\hline $\begin{array}{l}\text { Maximum dose } \\
\text { rate (kGy/s) }\end{array}$ & 10 & 30 & 60 \\
\hline $\begin{array}{l}\text { Processing unit } \\
\text { dimensions (m): }\end{array}$ & \multicolumn{2}{|c|}{$0.7 \times 0.7 \times 2$} & $1 \times 1.2 \times 2$ \\
\hline Water cooling & \multicolumn{3}{|c|}{ self-contained } \\
\hline
\end{tabular}

When developing the systems special consideration was given to the radiation safety. The radiation shielding is made of lead $10 \mathrm{~mm}$ thick and consists of two shells. The first shell (ref. 7) encloses the electron gun and the irradiation zone. The second one (ref. 8) encloses the electron gun, the irradiation zone, and the transformer. All joints have been developed very thoroughly. Many times repeated measurements of radiation around the PYXIS system show it is close to the background level.

The system is a computer-controlled unit. The system parameters vary within broad ranges. The operator can set the electron energy, pulse repetition rate, duration of irradiation or the radiation dose.

During the irradiation session the control apparatus performs measurements of every pulse amplitude and executes fast turn-off of high voltage and the electron beam in case of the breakdown. The computer provides for continuous monitoring of all major parameters of the system as well as diagnostics of possible malfunctions.

\section{CONCLUSIONS}

The irradiation systems presented have a compact design and provide for safe and reliable operation. The systems are simple in control and can be safely operated in any room. One of these systems has been in operation for more than 3 years now.

The systems can be very helpful in science of materials and used for material treatment, for surface radiation sterilization, for seeds disinfestations, etc.

\section{REFERENCES}

[1] S. Nablo, R. Cheever. Radiat. Phys. Chem., vol. 14, pp. 267-276, 1979.

[2] Z. Livne et al. Radiat. Phys. Chem., vol. 38, no 6, pp. 565-572, 1991.

[3] S. Krylov et al. Proc. of 1999 Particle Acc. Conf., pp. 2567-2569. 